\title{
Transformación de la prensa escrita en Neiva
}

\author{
Transformation of the press in Neiva
}

\section{Transformation del presse in Neiva}

\author{
Henry Rubiano Daza \\ Doctor en Ciencias Sociales y Humanas. \\ Universidad Cooperativa de Colombia. Neiva. Colombia.
}

\section{Resumen}

La prensa escrita en el siglo XX y lo corrido del presente en Neiva ha tenido una marcada relación con la política y la religión, por encima de lo corporativoempresarial; así se evidencia en el presente texto histórico de corte cualitativo-etnográfico, especialmente. Para el desarrollo de esta investigación se abordaron más de 30 fuentes, especialmente entrevistas a profundidad y el análisis documental buscando un análisis desde lo histórico social-político. El aporte a la historiografía, de este filón, está centrado en que producción informativa noticiosa de los medios está transversalizada por la autocensura, por aquello de la relación de las empresas mediáticas con la dirigencia política, los gremios de la producción y las entidades estatales.

Palabras claves: Producción informativa, autocensura, circulación, historiografía, Tic, Medios populares, periodismo digital.

\section{Zusammenfassung}

Die Presse im 20. Jahrhundert und die Begründung derselben in Neiva stehen in enger Verbindung mit der Politik und der Religion, ..., wie der vorliegende historische Text beweist. Für diese Untersuchung wurden mehr als 30 vor allem dokumentarische Quellen erforscht. Sie weisen darauf hin, dass die informative Nachrichtenproduktion der Medien durch die deutliche Verbindung der Medienunternehmen mit der politischen Führung, der Industrie und den Körperschaften des Staates in Verruf geraten ist, da die Medien von den Publikationsrichtlinien abhängig sind und somit ein hohes Niveau der Selbstzensur generieren.

Acercarnos a la historia de la evolución de la prensa escrita en Neiva desde el siglo pasado a lo corrido del presente, requiere más que una descripción, un análisis de sus transformaciones y de sus aporte a la construcción de región, toda vez que este modelo mediático permiten la trascendencia de los procesos comunicacionales en periferia, limitados por la distancia, el tiempo y su infraestructura de acceso.

Para este presente aporte historiográfico se abordaron

\section{Abstract}

The written press in Neiva, during the 20th century and part of the current century, has had a particular relationship with politics and religion, beyond the business realm. It is thus evidenced in the present historical, ethnographic and qualitative text. The development of this research study used more than 30 sources, especially rigorous interviews and documental analysis in search of a type of historical, social and political analysis.

Keywords: informative production, self-censorship, circulation, historiography, popular media, digital journalism.

1 - SÁNCHEZ R., FRANCISCO, "Periodismo en el Huila", en Revista Huila, Edi. Academia Huilense de Historia No. 31, 1994

2 - Autor de varios textos históricos. Actual presidente de la Academia Huilense de Historia.

3 - Comunicador Social y magíster en Historia de la Universidad Nacional. Es docente de la Universidad Surcolombiana y autor de ACFBEDO RESTREPO, JUAN CARLOS, El apetito de la injuria: libelo, censura eclesiástica y argumentación en la prensa del Huila (1905-1922), Neiva, Editorial Universidad Surcolombiana, 2008.

4 - Periodista e historiador. Autor del texto TRIILERAS, ROA ÁLVARO, Del linotipo al satélite: los medios de comunicación y el periodismo en el Huila, Neiva, s.n., 2005. 
En trabajo anterior, publicado en la Historia Comprehensiva de Neiva, dividía el análisis de la prensa escrita en tres periodos: el primero lo referenciaba entre 1900 y 1966, ciclo marcado por el bipartidismo conservador-liberal y el pensamiento religioso; el segundo entre 1966 y 1994 por haber evidenciado el nacimiento de número significativo de medios impresos, que si bien tenían la impronta de un campo específico -político, religioso, cultural o académico- empezaban a reflejar una identidad o estructura narrativa ligada a los géneros periodísticos; y el tercer periodo de 1994 a 2012, porque en él se observa la migración de la prensa escrita impresa a los medios digitales, considerados hoy como parte de la tendencia dominante en el mundo de la información periodística (Rubiano, 2013. P. 72). Estos ciclos categoriales permiten dar, en parte, respuesta a preguntas como ¿Cuál ha sido el aporte de la prensa escrita al desarrollo de ciudades emergentes como Neiva? o ¿Cómo ha evolucionado la calidad de la producción noticiosa en los medios impresos de Neiva?, este último cuestionamiento relacionado con la autocensura en el ejercicio del desarrollo de este oficio y más tarde profesión.

Sin duda, no se puede marginar de este filón temático el análisis del papel de la prensa en la conquista de libertades, la garantía de los derechos y la construcción colectiva de democracia, para darle paso a reflexionar sobre las transformaciones de las prácticas periodísticas con las tecnologías de la información y la comunicación (TIC), que como bien lo resalta Habermas este modelo, permiten espacios de opinión pública implantando la simultaneidad abstracta de una red virtualmente siempre presente de contenido de comunicación (Habermas, 2008. P 552).

En este análisis histórico es prudente recordar que hace más de cinco siglos la imprenta de tipos móviles, el invento de Johannes Gutenberg, revolucionó al mundo, a tal punto que se produjeron importantes cambios en la cultura y la sociedad y potenció profundamente la revolución de la escritura, la difusión de conocimiento contribuyendo al fortalecimiento de las revoluciones que condujeron a considerables transformaciones sociales y políticas. Gutenberg abrió las puertas a otra galaxia donde la imprenta se convirtió en una herramienta para el desarrollo social e intelectual. Gracias a la invención del alemán se puede dividir la historia del hombre en un antes y un después. Además que este nuevo artefacto hizo posible el acceso a la información y su masificación, lo que permite deducir que Gutenberg contribuyó también enormemente con el desarrollo del periodismo impreso, aunque este haya aparecido casi dos siglos después (Rubiano, 2013,P. 73)
Con este invento, podemos decir que el periodismo, como género o modelo informativo, arrancó a principios del siglo XVII en Europa Occidental con la impresión de los primeros periódicos producto del intercambio de noticias en escenarios conversacionales o lugares públicos, como los cafés londinenses. Muestra de ello es que para 1609 algunos impresores empezaron a recoger los comentarios, las discusiones políticas y las novedades que traían los marineros y las socializaban en estos sitios para imprimirlas en papel (Covach, 2004; pág 30). Con la evolución de los primeros periódicos, los políticos ingleses empezaron a hablar de un nuevo fenómeno al que denominaron opinión pública. Durante el siglo XVIII, algunos periodistas e impresores formularon por primera vez una teoría sobre las libertades de prensa y de expresión. Evidentemente, la historia de la prensa está marcada por una tendencia emancipadora en la cual se observa con frecuencia el poder que el ejercicio periodístico les ha dado a las comunidades para autogobernarse al permitir el acceso a la información al conocimiento- del mundo que las rodea.

Es claro que la prensa escrita tiene sus orígenes en la necesidad de informar, aunque en su forma primitiva no haya alcanzado las connotaciones sociales y democráticas que posee hoy. Sin embargo, la necesidad de información supone salir de la caverna, tal como lo mitificó Platón en su VII Libro la República, en donde plantea la situación del ser humano frente al conocimiento: el hombre ha tenido la continua necesidad de estar informado sobre su entorno y de lo que lo afecta e incluso, de servir como orientador de la realidad. Así lo sostiene también el historiador Mitchell Stephens, ${ }^{6}$ quien ha estudiado la forma en que la información ha influido en la historia. Para él y para varios estudiosos, las personas ansían las noticias por un deseo que se podría llamar "el instinto de estar informado"; la información permite acceder a lo desconocido, proporciona seguridad y permite planificar la vida. $^{7}$

Con este oficio, el periodismo, se han creado saberes que son practicados por los seres humanos desde que disponen de un lenguaje y una cultura colectiva. ${ }^{8} \mathrm{El}$ intercambio de información se ha convertido en la base a partir de la cual se crea comunidad y se conforman relaciones humanas, como las establecidas en las grandes civilizaciones -Grecia y Roma-. Estas convirtieron a la información en un vínculo social, en la forma de abordar los asuntos públicos y los intereses colectivos.

Lo relacionado anteriormente nos lleva a interpretar que la imprenta no sólo permitió la difusión de historias y de culturas; sino como arguye Darío

5 - KOVACH, BIL, Elementos del periodismo, Bogotá, Ediciones El País, 2004, pág. 30.

6 - STEPHENS, MITCHELL, A History of News: From the Drum to the Satellite, New York, Viking Penguin, 1988. Stephens es profesor de periodismo y comunicación de masas en la Universidad de Nueva York.

7 - STEPHENS, A History of News... pág. 29.

8 - LÉVY, PIERRE, "La inteligencia colectiva nuestra más grande riqueza". Consultado en: http://sociologiac.net/2007/07/19/pierre-levy-lainteligencia-colectiva-nuestra-mas-grande-riqueza/. Publicado originalmente en "L'intelligence collective, notre plus grande richesse", Le Monde, 24 de junio de 2007. 
Villanueva, ${ }^{9}$ superó la primera invención del hombre, el alfabeto fonético, pues con esta herramienta se reificó la palabra y, con ella, la actividad intelectual. ${ }^{10}$ El invento de Gutenberg marcó un hito fundamental en la historia de la comunicación humana y muchos historiadores ven en la imprenta el punto de partida de las transformaciones sociales y políticas de Europa y, desde luego, de los lugares a donde llegó su influencia como en Colombia y en zonas de periferia como Neiva.

La regularidad de las hojas noticiosas producidas a través de la imprenta posibilitó a finales del siglo XVI la aparición de las gacetas financiadas por iniciativa privada, formatos que hicieron el triunfo del periodismo impreso, que se consolidó fundamentalmente en Francia a lo largo del Siglo XVII. En el marco de la Revolución Francesa, las ideas se difundieron a través de libros, cartas, panfletos, libelos, semanarios y en los salones franceses. El público, cada vez más amplio y crítico, encuentra su refuerzo natural en una prensa cada vez más reflexiva e independiente. La prensa se convierte, por primera vez y de un modo propio, en el órgano crítico de un público en lo que más luego se referenciaría como el cuarto poder. Con la Revolución Francesa la libertad de expresión queda conquistada y la Declaración de los Derechos del Hombre y del Ciudadano consagra la libertad de opinión y de expresión. ${ }^{11}$

Años más tarde, en Colombia se editan las primeras hojas denominadas Aviso de un Terremoto, que para algunos historiadores son el inicio del periodismo en Colombia, y editadas en la imprenta de don Antonio Espinoza de los Monteros. Otros académicos e historiadores consideran al cubano Manuel del Socorro Rodríguez como el padre del periodismo colombiano, luego que él como bibliotecario sacara a la luz en 1791 el Papel periódico de la ciudad de Santa Fe de Bogotá, semanario que circuló entre 1791 y 1797 , y que alcanzó a publicar 265 números. Luego de estas publicaciones aparece la divulgación de los Derechos del hombre y el ciudadano de don Antonio Nariño, material impreso que fue relacionado como la piedra en el zapato para la Corona Española. De esta forma, la prensa escrita comienza a contribuir al proceso de emancipación política al informar a la opinión pública sobre los logros de las luchas europeas en la búsqueda de la igualdad y la defensa de los derechos del hombre y del ciudadano.

El periodismo se perfiló desde entonces como el símbolo de la libertad para los pueblos subyugados, no solo en Colombia sino en toda Latinoamérica. Es así como la historia del periodismo colombiano se confunde con la historia patria, especialmente en la evolución de las ideas políticas, desde su fundador Manuel del Socorro Rodríguez con su Papel Periódico de 1791, pasando por Francisco de Paula Santander y los próceres, hasta nuestros días. ${ }^{12}$

Año tras año los periódicos y las revistas han plasmado apasionadamente los preceptos y las ideas que provienen de la política y la religión. Dos siglos después empiezan a surgir empresas periodísticas de tradición como El Espectador, periódico político, literario, noticioso e industrial. Es importante referenciar que en el primer editorial Fidel Cano, su fundador, escribe: "Nos proponemos primeramente aprovechar en servicio del liberalismo como doctrina y como partido, la escasa suma de la libertad que a la imprenta le han dejado las nuevas instituciones, y luego contribuir y procurar que otros contribuyan al cultivo de la patria"."

De la misma forma que El Espectador, en los años siguientes se creó un buen número de periódicos cuyo contenidos se caracterizaban por tener un carácter de opinión política más que noticioso, sin dejar de lado la inclinación literaria que había guiado los pioneros del periodismo; esta era, sin duda, una buena mezcla de expresión intelectual. El periódico El Tiempo, hoy el más representativo del país según el Estudio General de Medios, fue fundado el 30 de enero de 1911 por Alfonso Villegas Restrepo y dos años más tarde fue adquirido por Eduardo Santos Montejo.

Con los años aparece una larga lista de los cuales se mencionan algunos en su orden El Colombiano de Medellín en 1912, Vanguardia Liberal de Bucaramanga en 1919, La Patria de Manizales en 1921, El Heraldo de Barranquilla en 1933, El Siglo en Bogotá en el año de 1936, El País de Cali en 1950, El Universal de Cartagena en 1961 y la lista continúa...

A mediados del siglo $\mathrm{XX}$, los dos diarios capitalinos más importantes -El Tiempo y El Espectador- vivieron la censura durante la dictadura de Rojas Pinilla, quien los clausuró; sin embargo, estos lograron seguir editándose con otros nombres. Sin duda este fue un periodo negro para la prensa en Colombia, pero permitió que se estructuraran las dinámicas del ejercicio periodístico.

El historiador huilense Camilo Francisco Salas realizó una importante clasificación del periodismo colombiano de acuerdo con las épocas y las tendencias que se manifestaron: en primer lugar, se parte del periodismo político del siglo XIX, relacionado directamente con la lucha revolucionaria y la formación de la República; en segundo lugar, se ubica el periodismo político y profesional de mediados del siglo $\mathrm{XIX}$, el cual tenía un carácter vocacional acentuado y estructurado en torno a la organización de los partidos políticos tradicionales. ${ }^{14}$

9 - VIILANUEVA, DARío, Después de la Galaxia Gutenberg y de la Galaxia McLuhan. After the Gutenberg Galaxy and the McLuhan Galaxy, Philadelphia, Society of Spanish and Spanish-American Studies, 2008.

10-VILLANUEVA, Después de la Galaxia.

12 - Cacua, Antonio. Historia del periodismo Colombiano. Bogotá. Ediciones Sua Ltda. II Edición. 1971.

13 - Cacua, Antonio. Historia del periodismo Colombiano. Bogotá. Ediciones Sua Ltda. II Edición. 1971.

14- Salas, Camilo F. Historia del periodismo huilense. Neiva, Edi. Instituto Huilense de Cultura. 1994. 


\section{Surgimiento de la prensa regional: de lo político a lo corporativo}

Una vez se instaló por ley la división del Estado Soberano del Tolima y la creación del departamento del Huila, territorio de la antigua provincia de Neiva, aparece una importante actividad político-administrativa que generó espacios de participación de la ciudad y de su entorno como departamento del Huila, especialmente a través de la prensa escrita. Estos formatos informativos se constituyeron a finales del siglo XIX y comienzos del XX en una de las formas más importante de relación social entre las regiones apartadas y el Estado. Delimiro Moreno ${ }^{15}$ referencia que la geografía infranqueable, la desarticulación económica y social, y el escaso desarrollo de las vías de comunicación no permitieron al grueso de la población trasladarse a otros lugares de la nación, e incluso, muchos de sus habitantes no viajaron más allá de los límites cantonales y regionales de sus lugares de origen durante toda su vida, lo que convertía a los medios informativos (la prensa escrita para esa época) en el único vehículo de la necesidad comunicativa.

La imprenta del departamento, de donde aparecieron las primeras ediciones de la Gaceta del Huila, fue creada poco después que el Departamento se instaurara como tal. Como era lógico esta empresa funcionaba en las mismas instalaciones de la Gobernación y tuvo una trayectoria significativa gracias a que las primeras publicaciones fueron las actas de la Asamblea y los documentos más importantes de la Administración Departamental.

De acuerdo con los trabajos históricos de Francisco Sánchez Ramírez "Periodismo en el Huila ${ }^{16 "}$, y de Camilo Franco Salas "Historia del periodismo huilense: la prensa escrita"17 , más este aporte histórico se tiene un estimativo de la fundación de 201 periódicos entre 1900 y el 2000 que tuvieron una marcada publicación, de los cuales una importante cantidad (49) eran de tendencia política. En su orden, le siguen las publicaciones de variedades con 32 periódicos; los informativos con 27 periódicos de diferentes tamaños, pero con cierta preeminencia del tabloide; las literarias e históricas con 19 periódicos; las institucionales con 17 y las culturales con 16. Con menor participación aparecen las publicaciones sindicales con 12; las comunales con 11; las de periódicos deportivos con 10 y otros con 8. Es necesario aclarar que, muy seguramente, existieron otros medios impresos en el sector educativo e institucional, pero por su carácter y reducida de circulación no tuvieron mayor trascendencia entre la comunidad huilense y neivana. (Ver siguiente gráfica y cuadro de principales impresos informativos)

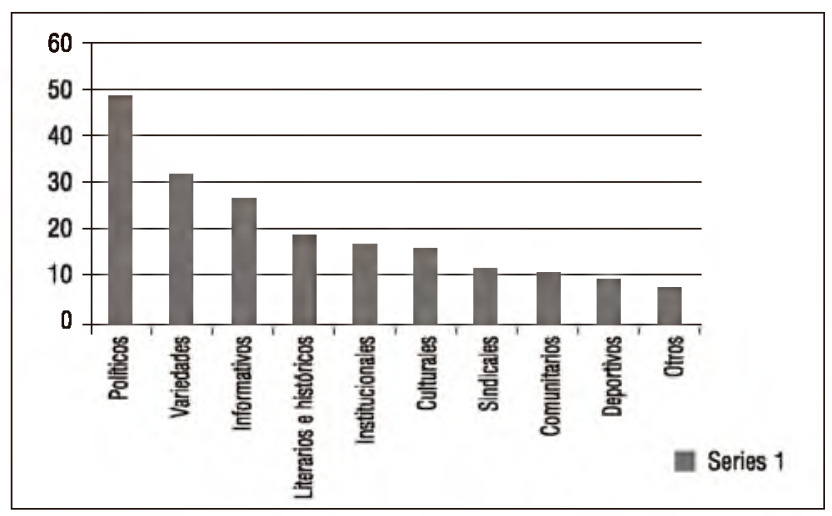

Fuente: Construcción propia

Esto demuestra que el desarrollo de la prensa escrita en el Huila, y especialmente en Neiva, contó con las mismas características de la prensa nacional, a raíz de la masificación de la información a través de las publicaciones nacionales y de la presencia de líderes políticos como propietarios o integrantes del equipo de redacción de los diarios. Entre ellos se destacan integrantes de comunidades eclesiásticas y alguna que otra familia de la élite, las que sabían que podían emplear los medios como un instrumento generador de dominio, rentabilidad y conservación eterna del poder político, marcando una estrecha relación de autocensura y colocando en serio peligro el buen ejercicio periodístico.

Los medios impresos de carácter político que nacieron a comienzos del Siglo XX con una tendencia hacia el centralismo y la integración nacional, marcada por la hegemonía de la prensa conservadora y clerical y la virtual extinción de la prensa de oposición. Como bien resalta Reynel Salas "hacia 1915 se inicia (...) un nuevo periodo, su característica primordial es la presencia de periódicos de tendencia liberal que surgen con el único propósito de hacerse voceros de sus ideas, agitar sus plataformas electorales y defenderse del desconocimiento a que los había sometido la prensa conservadora y católica". ${ }^{18}$

Juan Carlos Acebedo, con base en lo expuesto por Salas, señala que el periodo 1936-1965 se caracteriza por estar "entre la ideología de partido y los intereses de grupo". Para Salas, cita Acebedo, ${ }^{19}$ en este hito aparecen periódicos partidistas que "sin ablandar completamente su posición ideológica, comienzan a incorporar un lenguaje menos radical y se inclinan a incorporar en esas páginas nuevos elementos relacionados con el desarrollo regional".

En este texto se presentan algunos de los periódicos y revistas publicados en Neiva. Aunque lo que se comenta normalmente es que estos productos periodísticos

15 - Periodista e historiador antioqueño. Miembro de la Academia Huilense de Historia.

16- Sánchez R., Francisco. Periodismo en el Huila. En Revista Huila No. 31, Academia Huilense de Historia., 1984.

17 - Salas, Camilo F. Historia del periodismo huilense. Neiva, Edi. Instituto Huilense de Cultura.

18 - Salas, Camilo F. Trayectoria del periodismo huilense. En Historia General del Huila Volumen 5. Ed. Academia Huilense de Historia, Neiva, 1996, Pag. 98.

19 - Acebedo, J. Entre el vértigo y la memoria. Neiva - Huila. Editorial Universidad Surcolombiana. 2005. 
no se encuentran en archivo, he aquí una síntesis de los ejemplares que reposan en el Centro de Documentación de la Academia Huilense de Historia y otros que se obtuvieron por medio de una pesquisa propia.

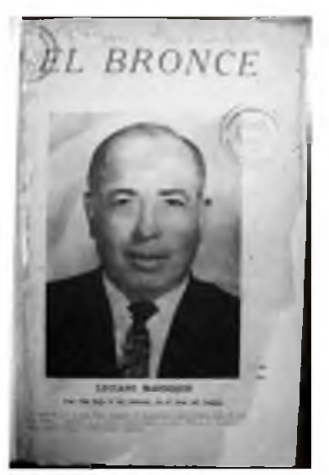

El Bronce, Neiva, N.o 7, 1 de agosto de 1965.

La revista El Bronce se consideraba como una publicación histórica que se sugería ser conservada como bien se expresa en uno de sus editoriales: "Conserve usted, lector, esta Revista en su biblioteca. Si la tiene. Si no la tiene, comience a formarla con ella. Con el tiempo aumentará su valía y significación bibliográfica. Porque página a página se escribe en ella la Historia del Huila". La línea editorial de este medio, dirigida por Augusto Ángel Santocoloma y de circulación bimensual y gratuita, se enfocaba en la publicación de textos literarios e históricos, a la vez que demostraba interés por la política y otros temas de interés general. Entre los autores que más participaban en sus páginas se encuentran Reinaldo Matiz, Amadeo Anzola, Alfredo Cedeño, Luis María Peña, Alberto Losada Lara, Gabriel Calderón, Régulo Suárez, Pedro Juan Navarro, Tulio Rubiano y Carlos Ortiz.
El semanario liberal El Debate fue fundado inicialmente en 1925. La edición a la que pertenece esta portada es de su segunda época y se publicó el 4 de agosto de 1962, cuando fue refundada por Tulio Rubiano con la dirección de Guillermo Plazas Alcid. Las instalaciones de este medio se encontraban en la

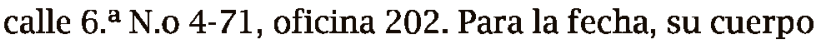
de redacción estaba conformado por Ignacio Solano Manrique, Hernando Emilio Cuéllar Lara y Fermín Segura Trujillo; Carlos J. Iriarte se desempeñaba como gerente del medio.

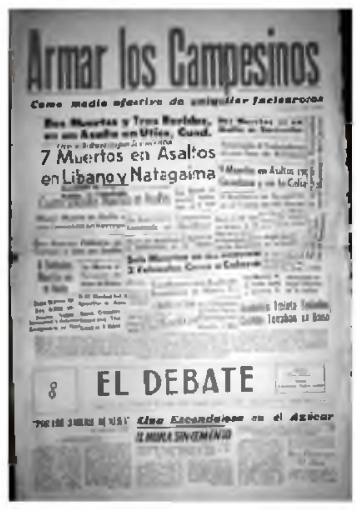

Edición de la segunda época de El Debate.

En el primer número de esta segunda época publicó un titular que rezaba: "Armar los campesinos como medio efectivo de aniquilar facinerosos [sic]", seguido de una serie de titulares de carácter judicial. En la parte inferior el contenido presentado está más relacionado con información social y económica. En relación a este medio, Camilo Salas planteó: "Históricamente este medio es considerado como la publicación periódica más prestigiosa que se haya hecho en el Huila, al menos de la prensa liberal". ${ }^{20}$

Cuadro 1. Principales impresos de circulación en Neiva (1966-2000)

\begin{tabular}{|l|l|l|l|}
\hline Nombre de publicación & Años circulación & Director & Orientación \\
\hline $\begin{array}{l}\text { Orientación Comunaly } \\
\text { Cooperativismo }\end{array}$ & $1966-1990$ & Jorge Arturo Calderón Cruz & Variedades \\
\hline Gremios y Personajes & 1967 & Fermín Segura T. & Variedades \\
\hline $\begin{array}{l}\text { Genealogias de la Provincia } \\
\text { de Neiva }\end{array}$ & 1967 & $\begin{array}{l}\text { Francisco de Paula Plazas } \\
\text { Sánchez }\end{array}$ & Cultural \\
\hline Impacto & 1968 & Ignacio Solano M. & Político \\
\hline $\begin{array}{l}\text { Anuario Estadistico del } \\
\text { Huila }\end{array}$ & $1968-1992$ & Directivos Planeación Dptal. & Órgano informativo \\
\hline El Deportista, 2. época & $1970-1972$ & Benhur Sánchez S. & Deportivo \\
\hline Vertiente & $1968-1971$ & Evaristo Cabrera Ch. & Político \\
\hline Me lo contó Mi Abuelo & 1969 & Saúl Perdomo Rivera & Novela \\
\hline Estampa Huilense & $1971-1974$ & Eduardo Araujo A. & Variedades \\
\hline Agrohuila & 1971 -actualidad & Félix Manrique P. & $\begin{array}{l}\text { Informativo } \\
\text { agropecuario }\end{array}$ \\
\hline Vanguardia Agraria & $1971-a c t u a l i d a d$ & Gabriel Molina C. & Variedades \\
\hline Pregón del Huila & $1973-1978$ & Marco Emilio Sánchez & Variedades \\
\hline $\begin{array}{l}\text { Próceres Huilenses en la } \\
\text { Independencia }\end{array}$ & 1974 & Eduardo Unda L. & Histórico \\
\hline
\end{tabular}

20 - Salas, Camilo F. Trayectoria del periodismo huilense. En Historia General del Huila Volumen 5. Ed. Academia Huilense de Historia, Neiva, 1996 , Pag. 133. 


\begin{tabular}{|c|c|c|c|}
\hline Nombre de publicación & Años circulación & Director & Orientación \\
\hline $\begin{array}{l}\text { Tierra de Promisión y otros } \\
\text { Poemas }\end{array}$ & 1974 & Luis Carlos Herrera. M.S.J. & Literario \\
\hline Neiva Progresa & 1974 & Arismendi Mora P. & Institucional \\
\hline Línea Liberal & 1975 & Arismendi Mora P. & Político \\
\hline Nueva Imagen & $1975-1984$ & $\begin{array}{l}\text { Catalina Muñoz y Orlando } \\
\text { Mosquera }\end{array}$ & Variedades \\
\hline Jacobo y sus Hijos & 1976 & Isabel Cabrera & Filosófico \\
\hline El Liberal Surcolombiano & 1977 & Guillermo Plazas Alcid & Político \\
\hline Enfoque Bambuquero & 1978 & Tirso Manuel Rubiano & Variedades \\
\hline Tribuna del Sur & 1978 & $\begin{array}{l}\text { Fernando Ospina H. y Delimiro } \\
\text { Moreno C. }\end{array}$ & Literario \\
\hline El Huila de Ayer & 1978 & Eduardo Unda L. & Histórico \\
\hline Notecoopsalud & 1978 & Ernesto Méndez C. & $\begin{array}{l}\text { Institucional } \\
\text { cooperativo }\end{array}$ \\
\hline El Huila para Usted & 1980 & Fermin Segura T. & \begin{tabular}{|l|} 
Variedades \\
\end{tabular} \\
\hline Morir de Pie & 1980 & Guillermo Bravo V. & Novela \\
\hline El Porvenir, 4. época & 1980 & $\begin{array}{l}\text { Misael Pastrana Borrero } \\
\text { (fundador) e Ismael Polanía T. }\end{array}$ & Político \\
\hline Contacto Liberal & & $\begin{array}{l}\text { Fundador: Julio Enrique Ortiz } \\
\text { Cuenca }\end{array}$ & Político \\
\hline $\begin{array}{l}\text { Quechuismos en algunas } \\
\text { regiones de Colombia }\end{array}$ & 1980 & $\begin{array}{l}\text { Eduardo Unda L. Gechem } \\
\text { Turbay }\end{array}$ & Cultural \\
\hline Meridiano del Sur & 1980-1989 & Fabio Echeverry C. & Variedades \\
\hline $\begin{array}{l}\text { Calendario Histórico del } \\
\text { Huila }\end{array}$ & 1981 & Miguel Ángel Díaz & Histórico \\
\hline Tribuna del Sur & 1981-actualidad & Liberio Jiménez J. & Variedades \\
\hline Revista Cifras & 1981-1991 & Directivos Contraloría Dptal. & Informativo \\
\hline Despertar Cooperativo & 1982 & Luis Humberto Manrique $\mathrm{B}$. & Variedades \\
\hline Ecos del Itre & 1982 & María de Jesús Artunduaga & Cultural \\
\hline Raspa & 1983 & Antonio Palomar A. & Literario \\
\hline El Mártir de Armero & 1982 & Pbro. Ignacio Córdoba & Histórico \\
\hline Monseñor Luis Calixto Leiva & 1983 & Pbro. Juan Bautista P. & Histórico \\
\hline Historial Cultural & 1983 & Yesid Morales R. & Cultural \\
\hline El Granjero & 1984 & Rodrigo Arias. Robert Salgado & Comunal \\
\hline Otro País & 1984 & Yesid Morales y Camilo Salas & Cultural \\
\hline Huila Laboral & 1984 & Gentil Fajardo & Laboral \\
\hline Megalitos & $1984-1986$ & Misael García & Cultural \\
\hline Eco-Impacto & 1985 & Guillermo Bravo Vega & \\
\hline El Mirador del Huila & $1985-1990$ & Camilo Francisco Sala & $\begin{array}{l}\text { Informativo } \\
\text { noticioso }\end{array}$ \\
\hline Magazín Mensaje & 1992-1999 & César Ramírez & Informativo \\
\hline $\begin{array}{l}\text { Revista Hogar Sagrada } \\
\text { Familia }\end{array}$ & 1984 & Sor María Raquel Escalante & Cultural \\
\hline La Nación & 1994-actualidad & Jesús Oviedo & $\begin{array}{l}\text { Informativo } \\
\text { noticioso }\end{array}$ \\
\hline
\end{tabular}




\section{Lo más representativo}

Entre 1966 y el año 1994 la prensa escrita en Neiva, con circulación regional y en algunos casos nacional, estuvo marcada por el auge de empresas periodísticas producto de inversiones de empresarios y gremios reconocidos en la región; estas empresas también manifestaban gran afinidad política con los partidos tradicionales, poniendo en peligro la independencia de los medios y de los periodistas y abriendo un amplio camino de autocensura. Entre los más representativos podemos contar cerca de 70 impresos entre periódicos y revistas que nacieron y circularon en Neiva en dicho periodo

Uno de los mayores analistas de los medios de información en todos sus formatos, ha sido Marshall McLuhan. Su texto "La comprensión de los Medios como las extensiones del hombre", publicado a mediados de la década de los sesenta del pasado siglo, permitió reflexionar sobre la importancia y transformación acelerada de estos órganos informativos en el desarrollo y construcción de sociedades. Casualmente en Colombia, en este periodo, emergieron un sinnúmero de medios regionales, especialmente impresos, que buscaban más que posicionar o hegemonizar una legado familiar, generar espacios para la construcción de ciudadanía y dimensionar el interés humano de quienes la conforman.

Para McLuhan la prensa es una forma confesional de grupos que proporciona participación comunal. Decía él a renglón seguido, en el texto referenciado anteriormente, que este tipo de medios puede dar color a los acontecimientos utilizándolos o dejando totalmente de utilizarlos, pero es la exposición comunal cotidiana de múltiples artículos o puntos de yuxtaposición lo que da a la prensa la compleja dimensión del hombre.

En esta investigación es válido presentar la experiencia de Julio Arturo Tovar, quien en 1940 fundó el diario mimeografiado vespertino "Notas del Día", con unos 100 ejemplares, considerado entre los historiadores de esta temática como Delimiro Moreno Calderón el primer periódico diario de esta zona del país, pese a su circulación duró poco menos de un año. Este medio fue un esfuerzo artesanal, obra de un solo hombre con su mimeógrafo, quien en las mañanas recorría la pequeña ciudad en busca de noticias y en la tarde distribuía su periódico con pocos avisos, que le permitían vivir y parte de los ingresos la dejaba para beber con sus amigos recibiendo los comentarios de su obra.

Doce años después, más exactamente el 4 de Diciembre de 1952 apareció otro impreso denominado "La Ciudad", que circuló con motivo del VIII Torneo Nacional de Baloncesto y fue dirigido por Alberto Yepes Trujillo. Este medio tuvo la colaboración de Franco Vargas Motta, abogado conservador y Gustavo Andrade Rivera, considerado el gran poeta y dramaturgo huilense. El periódico se editó diariamente, durante los quince días del campeonato en la Imprenta Departamental, informando los resultados de esta disciplina deportiva de la noche anterior.

Catorce años más tarde el médico Maximiliano Duque Gómez en la Hacienda Matamundo, donde hoy funciona un hotel con el mismo nombre, funda el Diario del Huila. Como Director de este medio nombró a su hijo el abogado Max Francisco Duque Palma. Su creador asumió las funciones de subdirector y gerente, mientras que Alfonso Camacho Leyva las de jefe de redacción. Diario del Huila salió a circulación el 8 de agosto de 1966 y su publicación fue considerada como un gran aporte al desarrollo del Huila, ya que estaba al nivel de otros editados en las principales ciudades del país. El primer número se editó con el eslogan "Por la libertad individual y el bienestar común informaremos del Huila para Colombia" y su primer editorial expresaba:

"En medio de grandes dificultades -casi invencibles- naturales en toda empresa periodística, y mucho más en un ambiente tan recortado y tímido como el nuestro, que por fortuna tiene que transformarse, como lo espera la nación y, sobre todo el departamento, aparece hoy a la luz pública el Diario del Huila. Editado en talleres y edificio propios, con capital y fuerza huilenses, su éxito se confía a la benevolencia con que lo acoja la crítica terrígena.

El periódico- intención poco menos que romántica- será una tribuna libre del pensamiento nacional pero especialmente del departamento, de los municipios y de sus gentes. Su altura moral y los principios filosóficos, religiosos, políticos y sociales que lo inspiran le aseguran una travesía de rectitud y serenidad invulnerables e inmodificables, así de procelosas sean las horas de suvida.

Lo entregamos con amor, entusiasmo y desinterés a la consideración de nuestros compatriotas en la esperanza en que sabrán recibirlo como parte de su patrimonio moral y espiritual y cuya dilapidación sería romper un grave compromiso con el esfuerzo común que debemos hacer en una de las horas más venturosas para nuestro desarrollo económico y social.

No en vano lleva el nombre de Diario del Huila que compromete el espíritu de colaboración y dinamismo de estas apartadas comarcas y constituye un reto a la solidaridad departamental, no por sus fundadores, sino por quienes hayan de seguir esta tarea de cultura y grandeza del departamento".

Los primeros números de este tabloide se editaron en una forma casi artesanal, toda vez que se imprimía con una técnica conocida como impresión al calor (letras fundidas en plomo y que con cada una de ellas armaba párrafos hasta completar el texto completo que llenaba las páginas). Año más tarde no sólo se transforma este medio al pasar al sistema offset, sino que su sede fue trasladada de la Hostería Matamundo al centro de la ciudad, dónde actualmente opera.

En 1974 las directivas del Diario del Huila nombran como jefe de redacción a Delimiro Moreno Calderón en medio de una crisis económica por sus escasos avisos publicitarios y una mínima impresión de 150 ejemplares, de los cuales devolvían 90. El trabajo periodístico de Moreno Calderón fue significativo, especialmente por el cubrimiento de la campaña presidencial de la cual salió triunfador el candidato liberal Alfonso López Michelsen, sobre el conservador Álvaro Gómez Hurtado y María Eugenia Rojas de Moreno, candidata de la ANAPO, así como el seguimiento al arribo a la gobernación del Huila de 
Olga Duque de Ospina. Este ejercicio periodístico, especialmente político, permitieron el renacer de este medio, toda vez que regresaron buenos columnistas y en poco tiempo este medio llegó a imprimir 1.500 ejemplares y a tener una mayor referencia y circulación. Pero como la dicha no es completa el incremento en la circulación aumentó los costos en papel, tinta y otros insumos, situación que contrastaba con el bajo ingreso por la publicidad, fuente fundamenta de sostenimiento de un medio. En esas condiciones, su único propietario, el abogado Max Francisco Duque Palma, cansado de subvencionarlo con los recursos que le dejaban sus haciendas, le propuso en abril de 1975 a Delimiro Moreno Calderón que se hiciera cargo de él. Ante este reto y sin un peso en su bolsillo Moreno Calderón buscó a los inversionistas Francisco Arias Silva, exgerente de Idehuila y a los esposos Fernando Ospina Hernández y Olga Duque. Una vez convencidos, fundaron la sociedad Editora del Sur la cual durante cuatro años (mayo de 1975 a mayo de 1979), lograron resucitar el Diario. En 1980 esta sociedad devolvió saneado a su propietario el Diario del Huila, sin quedarle debiendo un solo peso del arrendamiento (única utilidad que hasta entonces había dado el periódico), con una circulación ya de cerca de 5.000 ejemplares, una estructura empresarial de la que carecía antes y un notable prestigio local y nacional.

Con motivo de sus 30 años de existencia el Diario del Huila fue renovado el 8 agosto de 1996, pasó de tabloide a formato universal, con un diseño más ágil. Sus primeros números en el nuevo formato se caracterizaron por publicar titulares más cortos y con más espacio para las noticias y los reportajes. En cuanto al personal, se vinculó un grupo de comunicadores y periodistas recién graduados de pre-grado y capacitados en el periódico santandereano Vanguardia Liberal, entre ellos el hoy director Germán Hernández, Nelson Rojas Osorio, Martha Lucía Monsalve, Olmedo Polanco, Mildred Falquez, Patricia Sánchez, Ludiana Minú, Érica Manchola y Henry Rubiano Daza como el primer editor económico de este medio. El cambio fue liderado por el gerente Max Duque Rengifo, nieto de su fundador, avalado por su madre doña María Mercedes Rengifo de Duque, quien fungía como Directora de este medio.

Para este nuevo proceso nuevamente contrataron los servicios de Delimiro Moreno Calderón como jefe de redacción. Este cambio implicó una gran inversión económica, toda vez que se adquirió una rotativa de cuatro torres marca Harris, así como la compra de equipos de cómputo y la vinculación, además de los periodistas, de un cuerpo de asesores con trayectoria en estas empresas mediáticas, como el diseñador y caricaturista Vladimir Flórez conocido con el seudónimo de Vladdo.

Diez años más tarde, la crisis de los medios impresos en Colombia entre el 2005 y el 2007, generada por el aumento de sus costos de producción (especialmente del papel), afectó al Diario del Huila a tal punto que los dueños decidieron en noviembre de 2007 arrendar sus instalaciones al Grupo JOM, propietario del diario La Nación. Pese a que el contrato estaba firmado por seis años, las partes llegaron a un acuerdo y el Grupo JOM entregó el diario en diciembre de 2011. Durante estos cuatros años de arrendamiento Diario del Huila perdió presencia en la región, lo que se evidenció en los indicadores de lecturabilidad del Estudio General de Medios. Para los lectores de estos dos medios y en especial los suscriptores y anunciantes les resultaba difícil entender que un solo empresario fuera el propietario de los dos medios impresos más importantes, al tiempo de expresar que no existía una marcada diferencia informativa entre La Nación y el Diario del Huila y por consiguiente la publicidad solamente era contratada en uno de los dos medios.

Ante esta nueva crisis del Diario del Huila la familia Duque Rengifo decidió hacer una nueva inversión. El 15 de junio de 2012 cambió nuevamente su formato y diseño para convertirse en un tabloide moderno, ajustado a los estándares mundiales; estas transformaciones buscaban que Diario del Huila volviera a posicionarse en el mercado como el medio impreso más reconocido del sur del país. Para el nuevo tabloide fue contratado el diseñador Darío Forero, quien ha trabajado en los cambios de formatos de reconocidos medios nacionales como El Colombiano, El Espectador, la revista Cromos y El País de Cali, entre otros.

Hoy, después de 48 años de existencia, este medio cuenta con más de 9.000 mil suscriptores, una circulación significativa y rompiendo fronteras toda vez que se distribuye en Bogotá, Caquetá y Putumayo; además de una marcada cultura mediática digital, valor agregado de la participación del periodismo en la región, toda vez que su página web cuenta con un promedio de 25.000 visitas diarias.

En estos 48 años es necesario destacar la tarea cumplida, bajo la dirección de la familia Duque, por los periodistas que le han entregado su esfuerzo profesional, desde su primer jefe de redacción, Alfonso Camacho Leyva; el segundo, Leonel Fierro Trujillo (después destacado periodista de El Tiempo y otros medios nacionales); el inolvidable y eficaz Fermín Segura Trujillo, muchos años mano derecha de la familia Duque, hasta que con Jesús Oviedo fundó La Nación; Gustavo Hernández Riveros, excelente jefe de redacción y editorialista serio y sabio; Fabio Echeverri Campuzano, primer periodista huilense en ganar un premio Simón Bolívar; Pedro Bonilla Díaz, muchísimo tiempo excelente caricaturista y jefe de redacción; Alirio Ríos Osorio, Jonathan de la Sierra, editorialista y escritor; Rodrigo Sanabria Gutiérrez, gran informador; Alejandro Saavedra, el extraordinario reportero gráfico, y muchos otros profesionales de la comunicación que sería muy largo enumerar aquí, pero que han formado el capital intelectual, no por intangible menos valioso, del que hoy se enorgullece el periódico.

Este tabloide aún sigue siendo propiedad de la familia Duque Rengifo y a la fecha es administrado por María 
Pía Duque Rengifo; la dirección periodística está a cargo de Germán Hernández Vera, quien tiene claro que para que el Diario del Huila recupere el reconocimiento del que disfrutaba en décadas pasadas tiene que retornar a su línea editorial tradicional, la que manifestaba una marcada tendencia a cubrir las problemáticas de la comunidad y los temas de importancia para la región.

Por este motivo, y retomando a McLuhan, "la prensa es inseparable del proceso democrático"... además que repite la emoción que tenemos, cuando utilizamos la sagacidad y al hacerlo podemos llevar el mundo exterior al tejido de nuestro propio ser. Esta emoción o translación explica el por qué las personas desean servirse de estos formatos comunicativos.

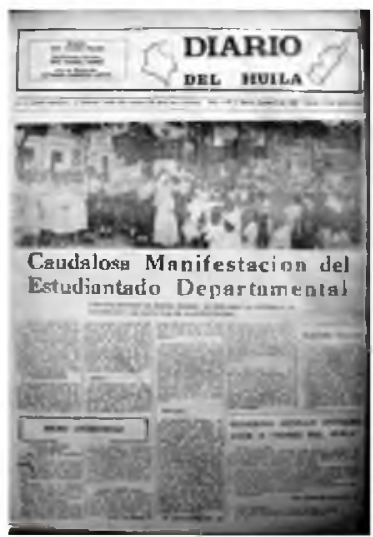

Edición del

Diario del Huila.

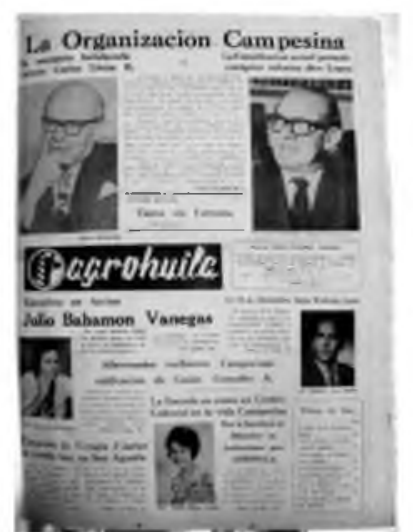

Edición de Agrohuila.
Otro medio impreso de importancia en la región ha sido el periódico fundado por Félix Manrique Agrohuila. Manrique adquirió la licencia de publicación el 28 de enero 1971, pero su primer número fue publicado el 11 de noviembre del mismo año con el eslogan: "Periódico al servicio del sector campesino de la región Surcolombiana". Este impreso fue uno de los más representativos de la reforma agraria que se deseaba implementar en el sur del país durante la presidencia de Carlos Lleras Restrepo, y a la fecha continua direccionado al sector agropecuario, lo que se evidencia en el contenido de sus notas y columnas.

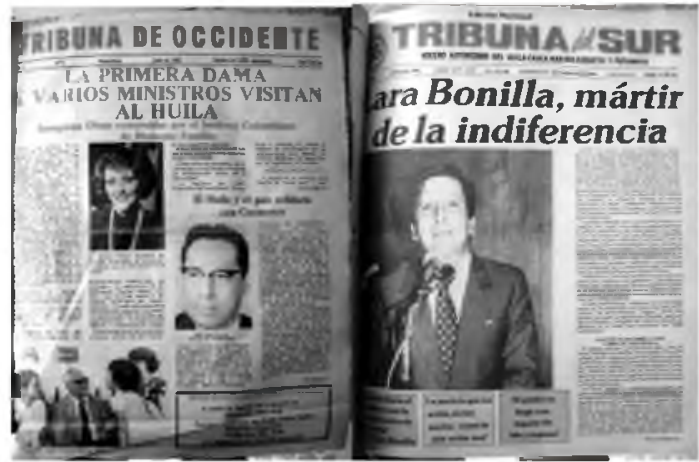

Ediciones de Tribuna de Occidente (julio de 1981) y Tribuna del Sur(mayo de 1984).
En el mes de marzo de 1981 sale a circulación Tribuna de Occidente, dirigido por Liberio Jiménez y con un marcado interés de publicar información relacionada con los municipios de La Plata y Nátaga, aunque funcionara en Neiva. Un par de años después, Tribuna de Occidente cambió de nombre y se convirtió en Tribuna del Sur; este medio despertó el interés del senador Rodrigo Lara Bonilla para convertirlo en su vocero en el sur del país, especialmente en los departamentos del Cauca, Nariño, Putumayo, Caquetá y Huila. El cambio de nombre y el alto contenido de opinión evidenciaron la marcada necesidad de expansión política del exministro de Justicia, quien fue asesinado por la mafia en 1984.

Al cierre del segundo semestre del 2012, Tribuna del Sur pasó a ser simplemente Tribuna. El cambio también fue notorio al mostrar una nueva política editorial marcada por la ecológica y enfocada a la protección de los recursos naturales de esta zona del país, especialmente del Macizo Colombiano y el río Magdalena.

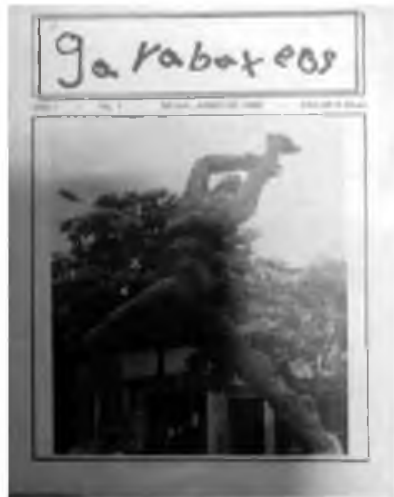

Primera edición de Garabatos, junio de1980.

La revista cultural y literaria Garabatos, adscrita a la Universidad Surcolombiana, se imprimía en los antiguos talleres litográficos de la institución y empezó a circular en junio de 1980. Entre sus responsables estaban Gustavo Briñez Villa, Luis Ernesto Lasso, Misael García García, Jorge Guebelly, Rafael Cortés Murcia, Leonel Arias, Miguel Perdomo Lince y Orlando Muñoz. En el editorial de su primer número se puede leer lo siguiente:

\begin{abstract}
Salimos en plena crisis universitaria, con reforma proimperialista por delante, pero con la seguridad de que tenemos la obligación de impulsar a las gentes jóvenes, a sus posiciones avanzadas en el terreno del Arte y la Literatura (...) No pretendemos competir con la revista institucional de la Universidad. Para que cesen celos y para que se exprese el pensamiento humanista de la Universidad y no solo el de Lingüística y Literatura- esperamos mantener, con la ayuda de todos los pensantes que se atreven a consignar su rebeldia y sus prospectaciones, esta publicación que bimensualmente irá por los colegios y del Departamento y de las Universidades que tengan carreras similares, con el propósito de intercambiar materiales y experiencias, para sentirse solidarios en las tareas de mantener el espíritu cada vez más cercado, más a punto de fenecer (...) 'Garabatos' -como su nombre lo indica- es para los que se inician en el serio trabajo de la creación poética, para los que investigan nuestro propio devenir cultural, pero fundamentalmente nace como necesidad de que expresen sus trabajos los estudiantes de los Talleres Literarios de la Surcolombiana (...). ${ }^{21}$
\end{abstract}

Pese a que el interés de Garabatos era fundamentalmente literario, también se caracterizaba por ser un medio de expresión que plasmaba una visión crítica de las políticas educativas -en especial de la superior-, al

21 - Garabatos, No. 1, junio de 1980. 
tiempo que publicaba permanentemente análisis de la coyuntura política tanto regional como nacional. Lamentablemente este medio circuló únicamente por un par de años.

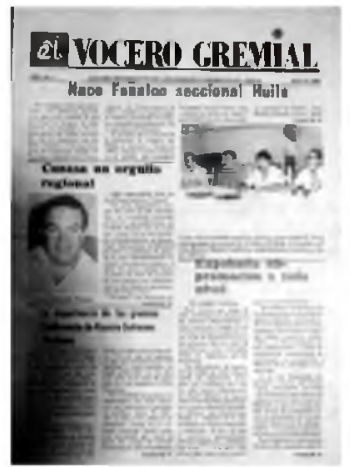

El Vocero Gremial, marzo de 1989.

El Vocero Gremial nació en marzo de 1989 como un espacio informativo de los gremios económicos del Huila, liderado especialmente por la Cámara de Comercio de Neiva. La dirección conjunta la ostentaban Arturo Hoyos Aristizábal, quien presidía la Asociación de Comerciantes de Neiva (ACON), y Ramiro Gutiérrez Perdomo, presidente ejecutivo de la Cámara de Comercio de Neiva. Su editor, Alberto Gómez Collazos, escribió en el primer editorial:

La Cámara de Comercio de Neiva y la Asociación de Comerciantes de Neiva, presentan hoy su órgano informativo ' $\mathrm{El}$ Vocero Gremial', el cual viene a llenar el vacío en nuestro medio, de la falta de un periódico especializado en las inquietudes y los temas de carácter gremial. La aspiración de sus directores no es otra diferente de lograr que los diversos sectores de la economía expresen sus criterios, sus problemas, sus soluciones y al mismo tiempo fijen su posición frente a los desafíos que conlleva el desarrollo de nuestro departamento y de Colombia. ${ }^{22}$

La Nación, impreso en la Editora Surcolombiana, se editó a partir del 15 de junio de 1994 como un proyecto empresarial que buscaba tener un impacto político local, por lo que dedicaba sus páginas centrales a las problemáticas de los barrios de Neiva y de algunos municipios cercanos. El diario salió a circulación con el eslogan "La noticia independiente". Su primer director fue Fermín Segura Trujillo y como de jefe de redacción se nombró a Carlos E. Salamanca Cerón, mientras que José Ignacio Ortiz Pascuas fue designado como gerente del medio.

Durante la etapa inicial de La Nación, uno de los integrantes de su equipo era la exministra de Educación Olga Duque de Ospina, quien años atrás hacía parte de la junta directiva del Diario del Huila. Llama la atención que esta dirigente política huilense de estirpe conservadora- hiciera alianza con el empresario Jesús Oviedo Pérez, presidente del Grupo JOM, hecho económico y empresarial que debilitó la imagen del Diario del Huila, toda vez que ella fue directora del Diario del Huila en los cuatro años que éste estuvo bajo arrendamiento de la Editora del Sur, pero del que nunca fue de la Junta Directiva con su propietario único, Max Francisco Duque Palma, primero y luego de su viuda y sus hijos.

Hoy en día La Nación es un diario regional de proyección nacional que circula en los departamentos del Huila, Caquetá, Putumayo, Amazonas, el sur del
Tolima e, incluso, Bogotá. Desde su primer número el diario se presenta como un periódico al servicio del interés colectivo y del desarrollo integral de la región, y como un aliado permanente de las acciones que promueven el progreso. En consecuencia, acoge todas las ideas expresadas con responsabilidad y serenidad dentro del marco de las instituciones democráticas del país.

En sus 18 años de existencia, La Nación ha logrado un sinnúmero de reconocimientos por su trabajo periodístico a nivel regional, especialmente los premios regionales de periodismo Reynaldo Matiz. De igual forma, fue especialmente relevante el hecho de que en el mes octubre de 2004 obtuviera el Premio Nacional de Periodismo Simón Bolívar en la categoría de mejor cubrimiento a una noticia, por el trabajo del periodista y hoy académico Henry Rubiano Daza, y en el que se denunciaban los malos manejos de los recursos del Plan Colombia durante la presidencia de Álvaro Uribe Vélez.

Recientemente, la Editora Surcolombiana se ha empeñado en estar a tono con los grandes periódicos nacionales y del mundo en lo referente a tener productos informativos especializados para los diversos sectores sociales, especialmente los noticiosos tradicionales y los populares. Para tal fïn, le compró en junio del año 2010 al diario La Patria de Manizales la rotativa de marca Harris V5 de ocho unidades, la cual cuenta con dos modernas empalmadoras que imprimen 25 mil ejemplares por hora en un solo tiraje; también adquirió un equipo de preprensa en línea CTP con un componente marcado de producción limpia de tintas ecológicas de última tecnología, muy similar al del periódico nacional El Tiempo. Con esta nueva infraestructura la Editora Surcolombiana no solo imprime el diario La Nación, sino que sacó al mercado dos nuevos productos: Óle -de tendencia popular- y Líder -dirigido al mercado del Caquetá-.

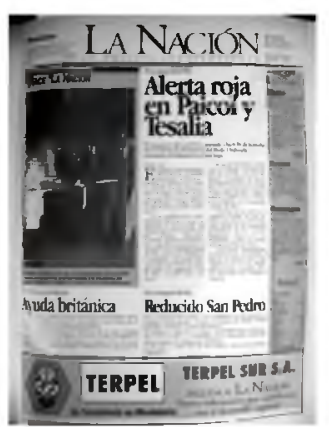

\section{Diarios populares}

En el mercado de los medios impresos ha emergido con fuerza los diarios populares. Estos órganos, según la Sociedad Interamericana de Prensa (SIP), están marcados por un diseño innovador y ágil, la publicación de noticias escuetas, un alto contenido de información judicial o "crónicas roja" y una comercializa-ción a un precio muy inferior al de los medios tradicionales existentes en el mercado. En este contexto nace en Neiva el 10 de marzo de 2010 el primer periódico popular denominado Extra, considerado un medio de crónica roja y que ha alcanzado gran aceptación en los estratos, uno dos y tres por el cubrimiento noticioso de los barrios populares. Este medio es del grupo editorial

22 - El Vocero Gremial, No. 1, marzo de 1989. 
El Periódico, cuyo representante legal es el industrial nariñense Hernando Suárez Burgos, propietario también del periódico Diario del Sur de Pasto y Diario Deportivo, El Periódico y Los Colombianos de Bogotá. Así mismo, este grupo editorial imprime el Extra de Bogotá, Extra de Kennedy y Extra de Soacha, así como otros 14 periódicos Extra en igual número de ciudades en el país.

Como respuesta a este estilo periodístico, el Grupo JOM, el mayor accionista de la Editora Surcolombia, fundó el 16 de diciembre de 2011 el diario óle, un tabloide con un tiraje inicial de 1.500 ejemplares. Para su editor general Fabián Hernández -quien desempeñó este mismo cargo en los inicios del periódico Extra de Neiva- Óle está orientado a la producción de noticias judiciales con una marcada redacción de estilo sensacionalista y crónica roja. También cuenta con una sección de notas comunitarias en la que priman las denuncias sobre la inseguridad en los barrios, los problemas de servicios públicos y los cuadros sociales marcados por la pobreza y el abandono de las instituciones del Estado.

\section{La prensa escrita en el nuevo ecosistema de la} información y la comunicación

Cuando se popularizó el internet en la última década del siglo XX, comenzó entonces una "invasión" de periódicos en la red. Sin duda, entre las empresas de comunicación la prensa es el formato que más se incorporó al ciberespacio, adaptando sus impresos a los formatos digitales. Los diarios, inicialmente los estadounidenses y europeos, concibieron la idea de hacer uso de los servicios on-line para plasmar sus contenidos en un nuevo soporte de una forma simple y económica; además, con esto participaban en un ámbito que pronto se convirtió en una fuente de información directa. Por supuesto, estos cambios también se evidencian en el mercado de los impresos del país. Los principales diarios colombianos como $E l$ Tiempo, El Espectador, El Colombiano, El Heraldo, El País, y La República, entre otros, ya poseen sus dominios en internet, teniendo en cuenta lo importante que resultar ser la participación en la web de los periódicos en el mundo periodístico.

En esta zona del país esta tendencia igualmente se hace presente. Es así como los dos medios impresos más importante, tanto el Diario del Huila como La Nación, hicieron su salto a la web, aunque de manera artesanal. A estos procesos también se le suman los medios alternativos de la misma tendencia digital, y entre los que se destacan opanoticas.com, tusemanario.com y suregion.com. Estos dos últimos aparecieron como resultado de ejercicios académicos de los programas de Comunicación Social de las universidades Surcolombiana y Cooperativa. La historia de estos medios en el formato digital se referencia al tener gran influencia en las dinámicas tradicionales de los medios, y dada la transición que vive la actividad periodística en el presente siglo, como bien lo afirma Alejandro Piscitelli al señalar que el internet es la imprenta del siglo XXI. ${ }^{23}$

\section{Periodismo digital}

De 1994 a 1995 se presentan una transformación en la práctica periodística en el Diario del Huila. Se pasa de las máquinas de escribir a los ordenadores Macintosh, muy apropiados en los medios impresos de gran escala y tiraje, al tiempo que en el país la cobertura del internet empezaba a crecer. De acuerdo con un trabajo de tesis de tres estudiantes de Comunicación Social de la Universidad Surcolombiana, ${ }^{24}$ con el objetivo de entregarle información de la región a los huilenses resientes en otros lugares del país y del mundo, y buscando aprovechar la oferta publicitaria que se puede hacer a través de internet, el Diario del Huila incursionó en el 2001, de forma paulatina, en este espacio mediático virtual con el dominio www.diariodelhuila.com.

Estos pasos son seguidos por La Nación, cuando su casa editorial empieza a mirar con otros ojos la importancia de crear un producto web utilizando la nueva tecnología. Si bien es cierto este medio había incursionado tímidamente con un blog, en el 2004 integró un diseñador y una plataforma web en el marco de la celebración de los 10 años del periódico. El 24 de junio de junio de 2004 hace el lanzamiento del portal noticioso www.lanacion.com.co, el cual impactó por su diseño innovador.

La cultura mediática digital permea a los nuevos profesionales, a tal punto que se genera un boom de internet y un desaforado uso de las TIC's como valor agregado de la participación del periodismo en la región. De manera empírica se presentan casos de creación de medios alternativos en la red, con lo que se busca proponer un periodismo diferente y llamativo para los habitantes de la región y los foráneos. La academia es la rampa que impulsa estos medios, ya que estudiantes y egresados incluyen en su proyecto de vida la elaboración de estos productos digitales con la recolección de información local, en un proceso que difiere al que desarrollan sus colegas impresos. La oferta que brinda la accesibilidad de los contenidos de la web es la mayor atracción.

De la dinámica académica en el programa de Comunicación Social y Periodismo de la Universidad Surcolombiana nace www.suregion.com, primera propuesta periodística digital diseñada para las necesidades del sur del país. Para ello, los productos periodísticos estaban enfocados en la premisa de crear reflexión y generar conocimientos en su población lectora; estas informaciones nacían de las experiencias académicas o

23 - Piscitelli, Alejandro. Internet la imprenta del siglo XXI. Barcelona. Ed. Gedisa. 2007.

24 - La tesis se titula: "Características del desarrollo del periodismo digital en el departamento del Huila entre el año 2004 -2010" y fue elaborada en el año 2010 por los estudiantes Leidy Lorena Pastrana Claros, Miller Eduardo Cortés Flórez y Andrés Felipe González Díaz. 
trabajos de clase dirigidos por el profesor Juan Carlos Acebedo. Sin embargo, los problemas de actualización de la información se convirtieron en obstáculos para el desarrollo periodístico de este medio digital. Su lecturabilidad estaba concentrada en la comunidad universitaria y sus temas reflejaban principalmente las preocupaciones relacionadas con la Universidad y se ocupaban muy poco de las problemáticas de Neiva. Al ser un medio que nació como un ejercicio de clase, su elaboración se limitaba al calendario académico de la Surcolombiana y durante las vacaciones no se publicaba información. Esta situación limitó la propuesta informativa y bajó el interés de lecturabilidad. Hoy en día www.suregion.com no aparece en la red.

Esta tendencia digital y el entusiasmo juvenil se conjugaron en "Un periodismo joven" (www.unperiodismojoven.com), propuesta que apareció en febrero del 2008 como un blog básico dirigido por Alejandro Cabrera Collazos, egresado del programa de Comunicación Social de la Universidad Cooperativa, sede Neiva. Cabrera, junto a un grupo de amigos, se dio a la tarea de generar una forma diferente de informar en un nuevo soporte mediático. Resultó difícil en sus comienzos posicionar este proyecto, debido a la limitada cobertura del internet en la ciudad hace algunos años. Sin embargo, este medio se fue abriendo paso a través de estrategias como la creación de bases de datos de correos electrónicos de habitantes de la ciudad para invitar a la comunidad a visitar su página y conocer la información publicada allí. En consecuencia, el número de visitas creció constantemente, lo que permitió la generación de más espacios dentro del mismo blog y facilitó su lecturabilidad. Después de esto, sus administradores decidieron optar por cambiar el nombre del dominio y darle paso a www.tusemanario.com, el cual genera más recordación entre los lectores. La venta de publicidad es su fuente de financiación, lo que ha permitido que esta propuesta digital continúe vigente.

Con un poco más de fuerza en el campo periodístico y bajo los lineamientos de periodismo digital se creó www.opanoticias.com, con el eslogan "Nuestra región minuto a minuto". Este medio está dirigido por Juan Carlos Charry González, director de la empresa de artes gráficas Sharry's Impresores, quien le apostó a la información instantánea que caracteriza al periodismo digital; a esto se le suma la experiencia de generar en formato físico un boletín de distribución gratuita como estrategia de comercialización, como lo suelen hacer los grandes diarios nacionales e internacionales. La idea ha resultado llamativa para la comunidad neivana y entre sus logros se destaca que en el 2010 obtuvo el premio regional de periodismo Reinaldo Matiz al mejor medio periodístico en internet.

Se debe destacar que uno de los fuertes de los medios digitales es su oferta multimedial, es decir, la posibilidad de incluir vídeos, audio, fotografías y demás aplicaciones. Estas opciones dan al medio un elemento extra de participación al usuario lector, quien avala la información o la refuta de acuerdo a su criterio.

Cuadro 2. Principales publicaciones en circulación en Neiva (2000-2012)

\begin{tabular}{|l|l|l|l|}
\hline Nombre de publicación & Años circulación & Director & Orientación \\
\hline El Huila en Blanco y Negro & 2002 -actualidad & César Ramírez & Revista informativa \\
\hline Opa Noticias & 2009 & Juan Carlos Charry & Informativo-noticioso \\
\hline Neiva Visible 2008 (Alcaldía) & 2008 & Héctor Aníbal Ramírez & Institucional \\
\hline Huila 15 días (Gobernación) & 2008 & Luis Jorge Sánchez García & Institucional \\
\hline El Amarrillo & 2009 & María Pía Duque Rengifo & Informativo noticioso \\
\hline Extra (Neiva) & 2010 & Fabián Hernández (editor) & Informativo noticioso \\
\hline Óle & 2011 & Fabián Hernández (editor) & Informativo noticioso \\
\hline
\end{tabular}

\section{Conclusiones}

Durante el siglo XX y lo corrido del XXI, la función social de la prensa escrita regional de ciudades capitales como Neiva es y seguirá siendo el puente para buscar alternativas de acercamiento entre lo público y lo privado, entre los social-comunitarito y el Estado; no obstante, su finalidad en el proceso de producción informativa noticiosa peligra por la marcada relación entre la dirigencia política y las entidades del Estado, así como por la cercanía con algunos gremios de la producción y las empresas de gran participación en el mercado local y nacional, toda vez que la existencia de estos medios está estrechamente relacionada a la pauta publicitaria que, a su vez, genera un alto nivel de autocensura. A pesar de esta situación de dependencia económica, los medios impresos en Neiva intentan sobrevivir y por momentos producen información que es resultado del periodismo investigativo o de denuncia en sus diferentes géneros o modelos periodísticos -de carácter social, económico, judicial y ambientaly que ha permitido llamar la atención de la opinión pública y evitar fenómenos de corrupción y violencia. 
Ahora bien, frente al futuro de los medios impresos se puede decir que mientras en muchos escenarios sociales se relaciona la muerte de los periódicos impresos con el periodismo digital o el "ciberperiodismo", la situación en Neiva, por ahora, es diferente. En Europa y en Norteamérica los medios impresos emergen a lo digital con nuevas prácticas periodísticas, modificado formatos (prensa, radio, TV, etc.) y creando nuevos relatos y escenarios informativos que son consumidos por diferentes sectores de recepción y por espacios académicos; por su parte, en regiones como el Huila, y en especial en capitales como Neiva, aparecen nuevas propuestas mediáticas porque aún hay una marcada brecha digital, toda vez que el acceso al servicio de internet y la adquisición de equipos de cómputo es limitado.

De igual forma, los grandes medios del país, e incluso los regionales, han definido en sus agendas noticiosas los temas de trascendencia nacional e internacional; no obstante, los lectores de los estratos bajos sienten preferencia por el contenido de los medios populares, que se han convertido en voceros de las comunidades, pese a que han circulado por poco tiempo. En ellos se relata su cotidianidad y su entorno social, por lo que cumplen la finalidad de la prensa regional o de periferia. Así se explica el éxito de los periódicos óle y Extra, el que espera también alcanzar un nuevo medio que sacará al mercado el Diario del Huilla. 